\title{
A comparative study of new-type-retractor-assisted wiltse approach TLIF, MIS-TLIF and traditional PLIF in the treatment of single- level lumbar degenerative diseases
}

HuanAn Liu

The Third Hospital of Hebei Medical University

JiaQi Li

The Third Hospital of Hebei Medical University

XianZheng Wang

The Third Hospital of Hebei Medical University

WeiJian Wang

The Third Hospital of Hebei Medical University

\section{YaPeng Sun}

The Third Hospital of Hebei Medical University

Lei Guo

The Third Hospital of Hebei Medical University

Fei Zhang

The Third Hospital of Hebei Medical University

Peng Zhang

The Third Hospital of Hebei Medical University

Wei Zhang ( $\square$ zworthopedics407@163.com)

The Third Hospital of Hebei Medical University

\section{Research Article}

Keywords: wiltse approach, MIS-TLIF, PLIF, lumbar degenerative disease, single-level, minimally invasive

Posted Date: November 18th, 2021

DOI: https://doi.org/10.21203/rs.3.rs-1069628/v1

License: (c) (1) This work is licensed under a Creative Commons Attribution 4.0 International License.

Read Full License 


\section{Abstract \\ Background}

To compare the clinical efficacy of new-type-retractor assisted wiltse approach Transforaminal lumbar Interbody Fusion (TLIF), Minimally Invasive Transforaminal Lumbar Interbody Fusion (MIS-TLIF) and traditional Posterior Lumbar Interbody Fusion (PLIF)in single-level lumbar degenerative diseases.

\section{Methods}

A retrospective study was conducted by analyzing clinical and imaging information of consecutive patients with single-level lumbar degenerative diseases who underwent either the new-type-retractor assisted wiltse approach TLIF or the MIS-TLIF or the traditional PLIF. 87 concurrent patients with similar age ,weight and severity of the imaging and symptom between June 2016 and December 2019 were included(wiltse approach 29 cases; MIS-TLIF 28 cases; PLIF 30 cases). The three groups were compared for perioperative indicators(including intraoperative blood loss, postoperative drainage volume, operation time, intraoperative fluoroscopy time, bed time), creatine kinase (CK), Visual Analogue Scale(VAS), Oswestry disability index (ODI), Japanese Orthopaedic Association (JOA) score, intervertebral fusion rate, muscle atrophy and fatty infiltration(including ratio of multifidus atrophy and ratio of lean-to-total cross sectional area(CSA)).

\section{Results}

Intraoperative blood loss, postoperative drainage volume and bed time in MIS-TILF group and wiltse group was significantly lower than PLIF group. The operative time and intraoperative radiation time of MIS-TILF group was significantly longer than wiltse group and PLIF group. CK level in wiltse and MISTILF group were significantly lower than PLIF group 1 and 3 days after surgery. PLIF group back pain VAS score was higher than wiltse and MIS-TLIF group. PLIF group displayed a higher ODI and a lower JOA score than the wiltse and MIS-TLIF group 6 months and 12 months after surgery. There was no significant statistical difference in lower limb pain VAS score and intervertebral fusion rate. Ratio of multifidus atrophy in the PLIF group was significantly higher than the wiltse group and the MIS-TILF group. Ratio of lean-to-total CSA (cross-sectional area) in PLIF group was lower than wiltse group and MIS-TLIF group after surgery. There was no significant difference between wiltse group and MIS-TLIF group except that MIS-TLIF group showed longer operation time and intraoperative fluoroscopy time while lower blood loss and drainage volume. 2 cases of durotomy and 2 cases of incision fat liquefaction occurred in PLIF group, 1 case of skin edge necrosis was found in MIS-TILF group. Patients were followed up at 3 months, 6 moyhs, 12 months after surgery, The mean follow-up time of the patients was $12.14 \pm 2.78$ months in wiltse group, $13.57 \pm 2.60$ months in MIS-TLIF group, and $12.73 \pm 2.80$ months in PLIF group, with no significant diffirence among 3 groups. (P凶0.05) 


\section{Conclusion}

Wiltse approach TLIF assisted with the new-type retractor is a more convenient and simple minimally invasive surgical method than traditional PLIF and MIS-TLIF, which requires a long learning curve, long operation and fluoroscopy time.

\section{Background}

With the development of minimally invasive spine surgery, surgeons can choose more minimally invasive surgery according to the patient's condition to reduce injury. Traditional open posterior surgery has become a commonly used surgical method for lumbar spine surgery due to its short learning period, sufficient decompression, wide applicability and reliable operation effect. However, owning to the large incision and extensive paravertebral muscles dissection, the innervation and blood supply of the multifidus muscle are greatly damaged during the posterior lumbar surgery, and many patients have intractable low back pain (LBP)after surgery, which seriously affects the quality of life of the patients. ${ }^{1}$ Therefore, how to reduce the incidence of complications including soft tissue injury and low back pain caused by surgery has become the focus of many surgeons. Lumbar surgery through the wiltse approach can significantly reduce damage to muscle and nerve. In 1968, Wiltse first described the paraspinal sacrospinalis-splitting approach to the lumbar spine. This approach was initially developed for fusion of spondylolisthesis. It allowed the surgeon to approach the area to be fused without cutting many of the supporting structures. ${ }^{2}$ In 1988 , Wiltse further described a posterolateral approach through the space of multifidus and longissimus to foramina for the treatment of far lateral disc herniation, spinal canal stenosis, and lumbar spondylolisthesis. ${ }^{3}$ Besides, wiltse approach has been used in fixation of vertebral such as vertebral fracture without neurological symptoms. Foley et al first published minimally invasive transforaminal fusion in 2002. ${ }^{4}$ Since its introduction, the MIS-TLIF has demonstrated to minimizing soft tissue disruption and minimizing destabilization of the spinal segment(s), thus leaving the smallest operative footprint possible while achieving the operative goal. ${ }^{5}$ Wiltse approach TLIF and MIS-TLIF are both minimally invasive surgical methods with the advantages of less bleeding, slight muscle injury, shorter hospital stay, and significantly reduced complications such as stubborn low back pain, providing more possibilities for reducing surgical injuries and complications in patients. ${ }^{6-8}$ However, MIS-TLIF still has the limit of long fluoroscopy time and long learning curve , as well as causing tissue trauma to some extent owing to the specific tubular compression to the muscle during the operation. ${ }^{9} \mathrm{In}$ this paper, firstly we showed a new type of retractor for wiltse TLIF. Secondly, a retrospective clinical study was conducted to compare the efficacy of new-type-retractor assisted wiltse approach TLIF, MIS-TLIF and traditional PLIF to identify their pros and cons. Thirdly, muscle atrophy and fatty infiltration were calculated in this research by measuring image of MRI by a quantitive way in order to clarify the degree of fatty infiltration.

\section{Materials And Methods}




\section{Inclusion and exclusion criteria}

Inclusion criteria: $₫$ a diagnosis of lumbar degenerative disease, including lumbar disc herniation with intervertebral instability, lumbar spinal stenosis, lumbar spondylolisthesis $₫$ with unilateral or bilateral lower limb symptoms (intermittent claudication or sciatica), after 3 months of conservative treatment, no obvious symptom relief was observed 『physical examination and imaging examination confirmed as a single level lumbar disease.

Exclusion criteria: $\mathbb{\otimes}$ lumbar spondylolisthesis $\geq 2$ degrees or accompanied by obvious

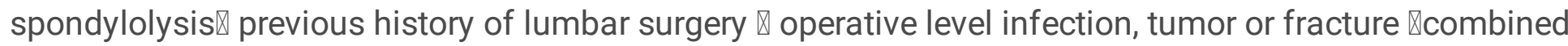
with other severe cardiovascular and cerebrovascular diseases or other surgical contraindications

\section{Baseline clinical data}

A total of 87 consecutive patients between June 2016 and December 2019 with lumbar degenerative diseases who had undergone wiltse approach TLIF(29 cases), MIS-TLIF(28 cases) or PLIF(30 cases) in our department were included. There were 35 patients (44.9\%) with unilateral lower limb pain, 29 patients (32.2\%) with lower limb pain and numbness, and 20 patients $(23.0 \%)$ with bilateral symptoms and 3 patients (3.4\%) with simple low back pain. Twenty-eight patients $(35.9 \%)$ presented unilateral or bilateral lower limb muscle strength decline to varying degrees. Cauda equina syndrome was present in 4 patients (4.6\%).

All the patients underwent lumbar radiograph of anterior and lateral position, hyperextension and hyperflexion position, lumbar intervertebral disc CT, and lumbar MRI before surgery. Among them, 15 patients were with lumbar spondylolisthesis and 21 patients were with lumbar instability. All patients' physical examination was consistent with imaging changes and all of them were confirmed as singlelevel degenerative diseases of the lumbar spine.

All patients in this study were informed and signed an informed consent, which was reviewed and approved by the ethics Committee of our hospital.

\section{Cases of group}

The patients were selected from the concurrent patients with similar age, weight and severity of the imaging and symptom, then were divided into 3 group according to the different treatment methods. Their baseline data showed no significant difference. Patients $(n=29)$ who had received wiltse approach TLIF operation were in the wiltse group. Patients $(n=28)$ who had received MIS-TILF (minimally invasive transforaminal lumbar interbody fusion) operation were in the MIS-TILF group. Patients $(n=30)$ who had received traditional PLIF (posterior lumbar interbody fusion) operation were in the PLIF group. All surgery were conducted by the same surgeon (Wei Zhang). 


\section{Surgical procedures}

\section{Wiltse approach TLIF ${ }^{10}$}

General anesthesia was used and patients were placed in prone position. (Step 1)Kirschner wire was used for positioning under c-arm X-ray fluoroscopy to determine the stage of responsibility. (Step 2) Bilateral access is provided thorough a midline skin incision with a length of about $8 \mathrm{~cm}$. The lumbar dorsal fascia was incised longitudinally at $2.5-3 \mathrm{~cm}$ from the posterior midline. Blunt dissection by the fingers were used to separate the medial multifidus from the lateral longissimus muscle. (Step 3) Identify the junction of the facet joints and the transverse processes, and pedicle screw was installed. Then the new type retractor was fixed on the pedicle screw (figure 5 and figure6) (Step 4). After full decompression of contralateral spinal canal and nerve root canal, the diseased intervertebral disc was resected, the cartilage endplate was scraped and bone fragments as well as intervertebral fusion cage were implanted into the intervertebral space. (Step 5) Finally, rod system was installed. (Step 6) One drainage tube was placed beside the incision. (Step 7)

\section{MIS-TILF (expandable tubular retractor assisted minimally invasive transforaminal interbody fusion $)^{5}$}

General anesthesia was used and patients were placed in prone position. (Step 1) The projection position of the adjacent pedicle of diseased segment were identified and marked by c-arm X-ray machine. With the assistance of fluoroscopy, percutaneous needle was used to locate the outer edge of each pedicle at the marking points respectively. (Step 2)Centering each puncture point, 4 transverse incisions about $1.5 \mathrm{~cm}$ in length were taken. The lumbar dorsal fascia was incised longitudinally. The space between the the medial multifidus and longissimus muscle was investigated and the articular facet joint were explored along this gap. After puncturing to the articular process with a puncture needle and inserting the guide wire along each puncture needle catheter, an expandable tubular retractor was inserted along the guide wire. The retractor was placed on the medial side of the articular process and on the upper margin of the intervertebral space in the responsible stage. (Step 3) After full decompression of spinal canal and nerve root canal, the diseased intervertebral disc was resected, the cartilage endplate was scraped and bone fragments and intervertebral fusion cage were implanted into the intervertebral space. If there were contralateral symptoms, the channel could be adjusted along the spinous process base to complete contralateral nerve root canal decompression, enlarge the contralateral nerve root canal and the central vertebral canal. After decompression and bone grafting, exit the working channel. (Step 4) Insert hollow pedicle screws into each pedicle along the guide wire. After the fluoroscopy position was satisfied, the pedicle screw rod system was installed. (Step 5) A drainage tube was placed through the incision on the decompression side. (Step 6) 


\section{Traditional PLIF}

General anesthesia was used and patients were placed in prone position. (Step 1) Under the perspective of C-arm X-ray machine, kirschner wire is used to locate and determine the responsibility stage. (Step 2) The posterior midline incision with a length of about $10 \mathrm{~cm}$ was taken. After the lumbar fascia was incised, the lateral paravertebral muscles along the spinous process are stripped to the bilateral facet joints. (Step 3) Then the pedicle screw rod system was installed. (Step 4) After full decompression of contralateral spinal canal and nerve root canal, the diseased intervertebral disc was resected, the cartilage endplate was scraped and bone fragments as well as intervertebral fusion cage were implanted into the intervertebral space. (Step 5) Finally, pedicle screw rod system was installed. (Step 6) One drainage tube was placed next to the incision. (Step 7)

\section{Outcome measures}

\section{Perioperative indicators}

Including intraoperative blood loss, postoperative drainage volume, operation time, fluoroscopy time, bed time.

\section{Indicators of muscle injury}

CK (Creatine kinase)

The serum CK level of patients were measured by enzyme coupling method before surgery, 1 day after surgery, 4 days after surgery, and 7 days after surgery, respectively, to evaluate the muscle injury intensity.

CK is the enzyme that catalyzes the reaction of creatine and adenosine triphosphate (ATP) to phosphocreatine and adenosine diphosphate (ADP). Serum creatine kinase (CK) concentrations have been used to investigate skeletal muscle injury caused by lumbar surgery. The normal CK level is considered to be 20 to $200 \mathrm{IU} / \mathrm{L}$. In this study, serum CK was measured using spectrometry.

\section{VAS (Visual analogue Score)}

The lower back pain score and lower limb pain score were evaluated at 3 days and 3, 6 and 12 months after surgery, respectively.

The Visual Analogue Scale (VAS)has been in use for the measurement of pain. It is a self-reported scale consisting of a horizontal or vertical line, usually 10 centimetres long $(100 \mathrm{~mm})$ with anchor descriptors 
such as (in the pain context) "no pain" and "worst pain imaginable" referring to the pain status. An introductory question (with or without a time recall period) asks the patient to tick the line on the point that best refers to his/her pain. VAS is feasible for clinical research and practice.

\section{ODI (Oswestry Disability Index)}

In this study, patients were surveyed in 9 aspects except sexual life.

The ODI were evaluated before surgery and 3, 6 and 12 months after surgery, respectively.

Oswestry disability index (ODI) is a principal condition-specific outcome measures used in the management of spinal disorders, and to assess patient progress in routine clinical practice. The ODI score system includes 10 sections: pain intensity, personal care, lifting, walking, sitting, standing, sleeping, sex life, social life and traveling. For each section of six statements the total score is 5 . Intervening statements are scored according to rank. If more than one box is marked in each section, take the highest score. If all10 sections are completed the score is calculated as follows: total scored out of total possible scorex 100 .If one section is missed (or not applicable) the score is calculated: (total score/( $5 \times$ number of questions answered) $) \times 100 \% .0 \%-20 \%$ is considered mild dysfunction, $21 \%-40 \%$ is moderate dysfunction, $41 \%-60 \%$ is severe dysfunction, and $61 \%-80 \%$ is considered as disability. For cases with score of $81 \%-100 \%$, either long-term bedridden, or exaggerating the impact of pain on their life.

\section{JOA score for low back pain}

The JOA score for low back pain were evaluated before surgery and 3, 6 and 12 months after surgery, respectively

The Japanese Orthopaedic Association score for back pain (JOA score) was established for evaluating LBP and/or lumbar spinal diseases and has been used to estimate the severity of LBP or clinical outcomes. The JOA score consists of four subscales: Subjective symptoms, Clinical signs, Activities of daily living and Urinary bladder function, providing clinicians with significant information. JOA score is 29 in total.

\section{Evaluation of intervertebral fusion}

In this study, two methods were used to evaluate intervertebral fusion, including lumbar radiograph of hyperextension and hyperflexion position and lumbar CT in extension position at the last follow-up. The radiographs were interpreted as showing incomplete union if there was mobility of more than $3^{\circ}$, a remaining clear zone, or no definite bone connection ${ }^{11}$.Lumbar CT scan in hyperextension is considered to be incomplete fusion if there is a gas pattern, a remaining clear zone, or no definite bone connection. ${ }^{12-}$ 


\section{Evaluation of muscle atrophy and fatty infiltration}

Lumbar MRI was used to evaluate the multifidus atrophy and fatty infiltration. The results, including the total cross-sectional area (CSA), lean CSA, ratio of lean-to-total CSA, were evaluated before surgery and at last follow-up after surgery according to the following methods. The CSA was measured at the operative level using the axial T2-weighted sequences with the RadiAnt DICOM Viewer software (Medixant. RadiAnt DICOM Viewer [Software]. Version 2020.2. Jul 19, 2020. URL: https://www.radiantviewer.com.).In order to identify the lean CSA, the region of interest (ROI) was drawn around the multifidus on both sides of the spinous process, excluding nearby fat, bone, and other tissues (Figure 1a). Ratio of multifidus atrophy = (preoperative CSA - postoperative CSA)/preoperative CSA. The ratio of lean-to-total CSA (Figure 1b) was utilized as an additional measurement of fatty infiltration as described in rotator cuff's research (ratio of lean-to-total CSA $=$ lean CSA /total CSA) $)^{15,16}$.

\section{Statistical method}

SPSS 20.0.0 statistical software was used for data analysis. For measurement data, statistical analysis were performed utilizing one-way ANOVA if they obeyed normal distribution and satisfied homogeneity test of variance, otherwise, nonparametric test of rank conversion is used. Counting data were compared by using The Chi-square test. The test level was 0.05 on both sides, and p-value of $\leq 0.05$ was considered statistically significant.

\section{Clinical Results}

\section{Follow-up time}

Patients were followed up at 3 months, 6 moyhs, 12 months after surgery, The mean follow-up time of the patients was $12.14 \pm 2.78$ months in wiltse group, $13.57 \pm 2.60$ months in MIS-TLIF group, and $12.73 \pm 2.80$ months in PLIF group, with no significant difference among 3 groups. (P凶0.05)

\section{The demographics}

Age, gender, the distribution of surgical segment, preoperative VAS score, preoperative ODI score and preoperative JOA score had no statistical difference(Table 1).

\section{Perioperative Metrics (Table 2)}

The 3 groups of surgery were completed by the same surgeon, and there was no surgical method change during operations. Intraoperative blood $\operatorname{loss}(\mathrm{F}=62.628, \mathrm{P}<0.001)$ and postoperative drainage 
volume $(F=72.048, P<0.001)$ were significantly different among the three groups. MIS-TILF group and wiltse group was significantly lower than PLIF group. MIS-TILF group was lower than that in wiltse group.

The operative time showed significant statistical differences among the three groups. The operative time of MIS-TILF group ( $169.11 \pm 29.38 \mathrm{~min}$ ) was significantly longer than that of wiltse group $(94.67 \pm 25.43 \mathrm{~min})$ and PLIF group $(98.97 \pm 24.98 \mathrm{~min})$. The fluoroscopy time of MIS-TILF group $(87.61 \pm 3.13 \mathrm{~s})$ was significantly longer than that of wiltse group $(22.41 \pm 0.73 \mathrm{~s})$ and PLIF group (22.41 $\pm 0.73 \mathrm{~s})$.

Postoperative bed time differed significantly among the three groups $\left(X^{2}=62.289 \llbracket P<0.001\right)$. The postoperative bed time of MIS-TILF group $(2.68 \pm 0.71 \mathrm{~d})$ and wiltse group $(3.3 \pm 0.48 \mathrm{~d})$ was significantly shorter than that of the open approach group $(5.13 \pm 0.90 \mathrm{~d})$. The postoperative bed time of MIS-TILF group was shorter than that of wiltse group.

\section{Evaluation of paravertebral muscle injury}

CK (creatine kinase) level showed no statistical difference at preoperative $(\mathrm{F}=0.307 \otimes \mathrm{P}=0.736)$ and 7 days after surgery $(\mathrm{F}=0.670, \mathrm{P}=0.515)$ among the three groups. There were statistically significant differences in CK levels 1 and 3 days after surgery $(F=9.331, P \llbracket 0.001)(F=15.967, P \llbracket 0.001)$. Wiltse and MIS-TILF group were significantly lower than PLIF group,while there was no statistically significant difference between MIS-TILF group and wiltse group in CK level 1 and 3 days after surgery $(P=0.907, P=0.860)$ (Table 3 \&Figure 2c).

\section{Low back pain VAS score and lower limb pain VAS score}

There was no significant difference in low back pain VAS score among the three groups before operation $(\mathrm{F}=0.350, \mathrm{P}=0.706)$. The back pain VAS score 7 days, 3 months, 6 months, 12 months after surgery showed significant statistical difference among 3 groups,and PLIF group back pain VAS score was higher than wiltse and MIS-TLIF group whereas there was no statistical difference among MIS-TLIF group and wiltse group. Back pain VAS scores were compared at different times within groups using analysis of variance for single factor repeated measurements. The results displayed that PLIF group back pain VAS score 7 days after surgery was $(1.4 \pm 0.388)$ higher than preoperation while MIS-TLIF and wiltse group showed no significant increase. 3 groups'back pain VAS score decreased gradually at 3 months, 6 months , 12 months after surgery.

There was no significant difference in lower limb pain VAS score among the three groups before surgery $(F=0.476, P=0.623)$ and 7 days $(F=0.586, P=0.559), 3$ months $(F=0.028, P=0.973), 6$ months $(F=0.828$, $\mathrm{P}=0.440), 12$ months after surgery $(\mathrm{F}=0.063, \mathrm{P}=0.939)$. (Table 4\& Figure $2 \mathrm{~d}$ and $2 \mathrm{e}$ ) 


\section{ODI (Oswestry Disability Index)}

There was no statistical difference in $\mathrm{ODI}$ among the three groups preopretion( $\mathrm{F}=0.422, \mathrm{P}=0.657)$ and 3 months after surgery $(F=0.230, P=0.795)$. However, there was statistically different 6 months $(F=3.282$, $P=0.042)$ and 12 month after surgery $(F=5.316, P=0.007)$, which manifested that PLIF group was higher than the wiltse and MIS-TLIF group. There was no statistical difference in ODI between the wiltse group and the MIS-TLIF group. ODI were compared at different times within groups using analysis of variance for single factor repeated measurements, and the results displayed 3 groups' ODI decreased gradually at 3 months, 6 months, 12 months after surgery. (Table 5\&Figure 2a)

\section{JOA score}

There was no significant difference in preoperative JOA score between the three groups $(F=0.09$, $\mathrm{P}=0.914)$. There were no statistically significant differences in JOA scores among MIS-

TLIF group, wiltse group and PLIF group 3 months after surgery $(F=2.383, P=0.098)$, while there were statistically significant differences 6 months and 12 months after surgery $(F=3.234, P=0.044)(F=3.874$, $\mathrm{P}=0.025$ ). The JOA score of PLIF group was lower than wiltse group and MIS-TLIF group 6 months and 12 months after surgery. There was no significant difference in JOA score between wiltse group and MISTLIF group at 6 months $(P=0.091)$ and 1 year $(P=0.827)$ after surgery. JOA score were compared at different times within groups using analysis of variance for single factor repeated measurements, the results displayed 3 groups' JOA score increased gradually at 3 months, 6 months, 12 months after surgery. (Table $5 \&$ Figure $2 b$ )

\section{The multifidus atrophy and fatty infiltration}

Ratio of multifidus atrophy in the PLIF group $(41.70 \pm 8.84 \%)$ was significantly higher than that in the wiltse group $(24.13 \pm 6.82 \%)$ and the MIS-TILF group (22.35 $\pm 5.03 \%)$, but there was no statistically significant difference between the wiltse group and the MIS-TILF group $(P=0.348)$ (Table 6). There were no statistically significant difference in ratio of lean-to-total CSA among the three groups before surgery $(F=0.749, P=0.476)$, while there were statistically significant differences after surgery $(F=8.852$, $\mathrm{P}<0.001)$,which in PLIF group(56.60 $\pm 7.52 \%$ ) was lower than in wiltse group (63.34 $\pm 7.74 \%$ ) and MIS-TLIF group $(64.03 \pm 7.19 \%$ )( Figure 3 ). There was no significant difference between wiltse group and MIS-TLIF group $(P=0.729)$.

\section{Intervertebral fusion rate}


The intervertebral fusion rate was $89.7 \%$ in wiltse group, $92.9 \%$ in MIS-TILF group and $93.3 \%$ in PLIF group (Figure 4). There was no significant statistical difference in follow-up time ( $F=1.975, P=0.145)$ and intervertebral fusion rate $\left(\chi^{2}=0.315, P=0.854\right)$.

\section{Surgical complications}

There were 2 cases of durotomy in the PLIF group, but no severe complication such as central system infection was found after complete suture and duraplasty. No dural rupture was found in wiltse group and MIS-TILF group. In the PLIF group, there were 2 cases of incision fat liquefaction, which were all healed after dressing change and debridement, while no fat liquefaction occurred in wiltse group and MIS-TILF group. In MIS-TILF group, 1 case of skin edge necrosis was found, which recovered after excision of skin edge suture. No instrumental failure and loosen was found in the three groups.

\section{Discussion}

Lumbar fusion can be accomplished in a number of ways, including traditional open PLIF surgery, MISTILF, wlitse approach TLIF, XLIF, and ALIF. The emergence of innovative surgical approaches has given clinicians more ways to reduce the operative trauma to patients, including excessive incision size, excessive blood loss and dural rupture risk, lumbar dorsal muscle atrophy and fatty infiltration, and intractable low back pain. Nevertheless, few studies concentrated on comparison of wlitse approach TLIF,MIS-TILF, and traditional PLIF concerning short-term clinical efficacy and long-term prognosis simultaneously. Furthermore, in this article, we showed a new type of retractor to wiltse TLIF designed by us, which could assist to retract the paravertebral muscles and expose the operating field. We found that despite the reduction in soft tissue damage囚MIS-TLIF still cause muscle trauma to some extent owing to the continuous compression to the paraspinal muscle by the expandable tubular retractor. Thirdly,muscle muscle atrophy and fatty infiltration were calculated in this research by measuring image of MRI by a quantitive way in order to clarify the degree of fatty infiltration. This article was to identify wiltse approach TLIF is more suitable for lumbar surgery than MIS-TLIF when surgeons need to reduce muscle damage as well as increase convenience in the surgery.

\section{Wiltse approach TLIF and MIS-TLIF®2 minimally invasive ways}

Wiltse approach TLIF and MIS-TLIF have the advantages of small incision, less multifidus injury and quick postoperative recovery.17In this study, wiltse group and MIS-TILF group significantly reduced intraoperative blood loss, postoperative drainage volume, and postoperative bed time(Table 2), which is consistent with previous studies of Lee et al. ${ }^{18} \mathrm{This}$ is mainly due to different surgical approaches.The posterior paraspinal muscles are mainly composed of multifidus, longissimus, and iliocostalis. Lumbar multifidus is an important muscle for lumbar segmental instability. Medial branch of dorsal rami innervates the fascicles of multifidus attached to the spinous process and plays an important role in maintaining lumbar segmental stability ${ }^{19}$. Extensive multifidus muscles stripping and retraction, damage 
to the dorsal rami of the posterior branches posterior lamina decompression caused by traditional posterior surgery will inevitably lead to increased intraoperative bleeding and more serious tissue damage, resulting in increased postoperative drainage volume and prolonged bed time, atrophy of multifidus muscles and chronic low back pain (LBP). On the contrary, wiltse approach TLIF and MIS-TILF reach the surgical site through the natural space between the multifidus and the longus muscle. Blunt muscle separation or tubular expansion can avoid direct cutting damage to the muscle tissue, which is more in accord with the concept of minimally invasive, and avoid related complications caused by prolonged bed stay, such as deep vein thrombosis of lower limbs and hypostatic pneumonia. Moreover, the intraoperative blood loss and postoperative drainage volume in MIS-TILF group were less than those in wiltse group.MIS-TILF has shown to reduce surgically related bleeding to a greater extent.

\section{Clinical simplicity and convenience: new-retractor-assisted Wiltse approach TLIF is better than MIS-TILF}

However, the operating and fluoroscopy time of MIS-TILF was significantly longer than that of wiltse approach TLIF and traditional PLIF, which was consistent with the study reported by Phan et al 20 . We analyze the possible reason as follow : Minimally invasive exposures are limited to the area of surgical interest and certain key anatomic landmarks within this limited field of view, that is, the surgeon need a longer learning curve to familiar with the anatomy to safely perform the procedure without exposing structures that are not being surgically treated. In order to ensure the correct position of working tubular and excise insertion of pedicle screw, long time of fluoroscopy is inevitable. In our study ,we found the fluoroscopy time of MIS-TLIF is 3 times longer than wiltse approach TLIF , and the operation time is about 60 minutes longer(Table 2). Traditional wiltse approach TLIF procedures use conventional lumbar surgery retractor, which is not convenient and increasing the difficulty of operation. The new type retractor can be fixed on the pedicle screw and facilitates exposure, by adjusting the retractor blade, the junction of the facet joint and the transverse processes can be identified easily in wlitse TLIF(Figure 5 and 6). Excision of hyperplastic ligamentum flavum and degenerative facet, removal of disc, internal fixation and interbody fusion can be performed under intuitive and clear surgical vision. The operation is convenient, safe and reliable, with sufficient decompression of vertebral canal and reliable clinical efficacy. From the aspect of simplicity and convenience combined with reducing radiation to patients and doctors and nurses, wlitse approach TLIF is more appropriate than MIS-TLIF.

\section{Muscle injury of wiltse approach TLIF and MIS-TLIF}

Postoperative CK level can be used as an indicator of muscle injury. ${ }^{21-23}$ Dapeng Zhang et al showed a significantly lower CK in the MIS-TLIF group vs PLIF after surgery $\triangle \mathrm{P} \otimes 0.001 \mathbb{2}^{24}$. In this study, CK level of wiltse group and MIS-TILF group was significantly lower than that of PLIF group on day 1 and day 3 after surgery, which proved that the degree of muscle injury during wlitse-TLIF and MIS-TILF was significantly lower than that during PLIF surgery. It's worth noting that no significant difference was observed between wiltse approach TLIF and MIS-TLIF(Table 3 and Figure 2c), which meant MIS-TLIF produced a similar degree of muscle damage comparing with wiltse approach TLIF. In a wiltse approach TLIF, the medial multifidus is separated from the lateral longissimus muscle using blunt dissection easily since there is a 
natural gap between the multifidus and longissimus muscle. The exposure process won't cause distraction of muscle fibers or overstretching, which conforms to the concept of minimally invasive. In a MIS-TLIF surgery, surgeon need to adjust the direction of the expandable tubular retractor to achieve adequate spinal decompression especially contralateral decompression. In this process, the compression to the paravertebral muscles is relatively more severe. Combined with the long operation time, the degree of muscle damage caused by the MIS-TLIF was not significantly lower than wiltse approach TLIF.

\section{Clinical efficacy : wiltse approach TLIF and MIS-TLIF are better than PLIF}

Cheng et al found that wiltse approach VAS of back pain at both 7 days and 3 months showed better results $(P<0.05)$ and VAS of leg pain showed better results in 3 months but had no significant difference in 7 days vs the traditional approach. ${ }^{25}$ In this research,7 days, 3 months, 6 months, 12 months after surgery back pain VAS score in MIS-TILF group, wiltse group was obviously less than PLIF group.VAS score of leg pain showed no statistical difference among the three groups. The difference with Cheng et al may origined from error of subjective assessment accompanied by VAS.PLIF group had a higher ODI a lower JOA score 6 months and 12 months after operation than MIS-TLIF group and wiltse group. The difference of ODI score, JOA score among the 3 groups was mainly related to postoperative long-term LBP derived from PLIF method.The lower extremity neurological symptoms of patients in each group were well relieved, indicating that wiltse approach TLIF and MIS-TLIF could achieve satisfying decompression effect in parallel with PLIF surgery. There was no statistically significant difference in intervertebral fusion rates among the three groups,which indicated that all the three surgical methods could achieve the expected fusion results.In conclusion, wiltse approach TLIF and MIS-TLIF can effectively alleviate back pain on the basis of relieving neurological symptoms, which are better than PLIF.

\section{Muscle atrophy and fatty infiltration by measuring image of MRl: a quantitive way}

Long-term effects of paravertebral muscle can be evaluated by MRI. Reduction of paravertebral crosssectional area and infiltration of fat and connective tissue are mainly manifested by enhanced signals on T2-weighted imaging. ${ }^{26}$ Junhui et al found that multifidus CSA at final follow-up MRI was significantly less than in wiltse group (CSA decreased by $7.6 \%$ ) than PLIF group( CSA decreased by $35.4 \%$ ). ${ }^{25}$ In line with Junhui, this study found the ratio of multifidus atrophy was significantly lower in wiltse and MISTILF group than PLIF group, while the difference between wiltse group and MIS-TILF group was not obvious(Table 6 and Figure 7). Ratio of lean-to-total CSA was able to quantitatively compare the degree of fatty infiltration. The results found that PLIF group was significantly lower than wiltse group and MISTILF group, while there was no significant difference between wiltse group and MIS-TILF group,(Table 6 and Figure 7)which further proved from the imaging perspective that the wiltse approach reduced the degree of multifidus muscle atrophy and retained more paravertebral muscle function compared with traditional PLIF surgery, which was helpful to maintain the spine stability.There was no statistical difference between wiltse group and MIS-TILF group, indicating that these two surgical methods could achieve similar surgical effects in reducing paravertebral muscle injury. 


\section{Complications}

Complications of intraoperative dural rupture and incision fat liquefaction occurred in the PLIF group, while no similar complication occurred in the wiltse group and MIS-TILF group. There was 1 case of skin edge necrosis in MIS-TILF group, but none in the other two groups. Wiltse approach TLIF and MIS-TILF has small incision and quick healing, which is beneficial to reduce the occurrence of postoperative complications. The occurrence of skin edge necrosis in MIS-TILF group may be caused by the unskillful surgical technique in early surgery, long operation time, and long compression time of fixed pipes on the skin, and no skin edge necrosis occurred after proficient surgery and shortened operation time.

\section{Limitations}

There are still some deficiencies in this study. First of all, this study is a retrospective comparative study, and the number of cases is relatively small. Secondly, the follow-up time of patients is still short, and there is a lack of statistics and comparison of longer term complications and surgical efficacy.Thirdly, merely the patients with single-level lumbar spine surgery were analyzed and all of them were L4-L5 and L5-S1 disc level lesions.Further research for patients with multi-segment and higher level disc degenerative diseases are necessary.Finally, the comparison of paravertebral muscle atrophy in this study was limited to MRI image evaluation. Relevant studies have shown that the results of pathology and electrophysiological assessment can further clarify the effects of different surgical procedures on paravertebral muscles from different perspectives. ${ }^{27}$ The author believes that the advantages and disadvantages of MIS-TILF and wiltse approach TLIF can be further identified through randomized controlled trials, multi-center, long-term follow-up, the inclusion of more patients with multi-segmental and intervertebral disc degenerative disease in higher level, and the inclusion of more evaluation indicators, such as paravertebral muscle tissue pathology and paravertebral muscle electrophysiological analyses.

\section{Conclusion}

MIS-TLIF, wiltse approach TLIF and PLIF can achieve satisfying surgical efficacy on single-level degenerative diseases of the lumbar spine in well selected patients, while MIS-TLIF and wiltse approach TLIF can significantly reduce bleeding, bed time, muscle injury, and low back pain, paravertebral muscle atrophy and fatty infiltration, which are superior to PLIF surgery. Though MIS-TLIF had less bleeding than wiltse approach, however it showed a paralleling degree of muscle trauma to wiltse approach TLIF. Considering the long learning curve, long operation and fluoroscopy time of MIS-TLIF, wiltse approach TLIF assisted with the new-type retractor is a more convenient and simple minimally invasive surgical method than MIS-TLIF.

\section{Abbreviations}

TLIF

Transforaminal lumbar Interbody Fusion 
MIS-TLIF

Minimally Invasive Transforaminal Lumbar Interbody Fusion

PLIF

Posterior Lumbar Interbody Fusion

CK

Creatine kinase

VAS

Visual Analogue Scale

ODI

Oswestry disability index

JOA

Japanese Orthopaedic Association

CSA

Cross sectional area

ATP

Adenosine triphosphate

ADP

Adenosine diphosphate

\section{Declarations}

\section{Ethics approval and consent to participate}

This study was approved by the ethics committee of The Third Hospital of Hebei Medical University.

\section{Consent for publication}

Not applicable

\section{Availability of data and materials}

All data supporting the findings are within the manuscript.

\section{Competing interests}

Authors declare no conflict of interest nor competing interest in relation to this study.

\section{Funding}

No funding was received in relation to this study.

\section{Availability of data and materials statement}

All data generated or analysed during this study are included in this published article [and its supplementary information files].

\section{Authors' contributions}


$\mathrm{HL}, \mathrm{WZ}$, and $\mathrm{JL}$ were involved in the study design, data acquisition, data analysis and interpretation and drafting. XW and WW were involved in the study design, data analysis, interpretation and drafting. YS, LG, FZ, and PZ were involved in the study design, data analysis. All authors read and approved the final manuscript.

\section{Acknowledgement}

Not applicable.

\section{Availability of data and materials}

All data supporting the findings are within the manuscript.

\section{References}

1. Onesti ST. Failed back syndrome. Neurologist. 2004;10(5):259-264. doi:10.1097/01.nrl.0000138733.09406.39

2. Wiltse LL, Bateman JG, Hutchinson RH, Nelson WE. The paraspinal sacrospinalis-splitting approach to the lumbar spine. J Bone Joint Surg Am. 1968;50(5):919-926.

3. Wiltse LL, Spencer CW. New uses and refinements of the paraspinal approach to the lumbar spine. Spine (Phila Pa 1976). 1988;13(6):696-706.

4. Foley KT, Holly LT, Schwender JD. Minimally invasive lumbar fusion. Spine (Phila Pa 1976). 2003;28(15 Suppl):S26-S35.

5. Lener S, Wipplinger C, Hernandez RN, et al. Defining the MIS-TLIF: A Systematic Review of Techniques and Technologies Used by Surgeons Worldwide. Global Spine J. 2020;10(2 Suppl):151S$167 \mathrm{~S}$.

6. Patel AA, Zfass-Mendez M, Lebwohl NH, et al. Minimally Invasive Versus Open Lumbar Fusion: A Comparison of Blood Loss, Surgical Complications, and Hospital Course. lowa Orthop J. 2015;35:130-134.

7. Isaacs RE, Podichetty VK, Santiago P, et al. Minimally invasive microendoscopy-assisted transforaminal lumbar interbody fusion with instrumentation. J Neurosurg Spine. 2005;3(2):98-105. doi:10.3171/spi.2005.3.2.0098

8. Park Y, Ha JW. Comparison of one-level posterior lumbar interbody fusion performed with a minimally invasive approach or a traditional open approach. Spine (Phila Pa 1976). 2007;32(5):537543.

9. McClelland S 3rd, Goldstein JA. Minimally Invasive versus Open Spine Surgery: What Does the Best Evidence Tell Us?. J Neurosci Rural Pract. 2017;8(2):194-198. doi:10.4103/jnrp.jnrp_472_16

10. Guiroy A, Sícoli A, Masanés NG, Ciancio AM, Gagliardi M, Falavigna A. How to perform the Wiltse posterolateral spinal approach: Technical note. Surg Neurol Int. 2018;9:38. Published 2018 Feb 14. doi:10.4103/sni.sni_344_17 
11. Frymoyer JW, Matteri RE, Hanley EN, Kuhlmann D, Howe J. Failed lumbar disc surgery requiring second operation. A long-term follow-up study. Spine (Phila Pa 1976). 1978;3(1):7-11. doi:10.1097/00007632-197803000-00002

12. Cook SD, Patron LP, Christakis PM, Bailey KJ, Banta C, Glazer PA. Comparison of methods for determining the presence and extent of anterior lumbar interbody fusion. Spine (Phila Pa 1976). 2004;29(10):1118-1123. doi:10.1097/00007632-200405150-00013

13. Nakashima H, Yukawa $Y$, Ito $K$, et al. Extension CT scan: its suitability for assessing fusion after posterior lumbar interbody fusion. Eur Spine J. 2011;20(9):1496-1502. doi:10.1007/s00586-011$1739-0$

14. Kitchen D, Rao PJ, Zotti M, et al. Fusion Assessment by MRI in Comparison With CT in Anterior Lumbar Interbody Fusion: A Prospective Study. Global Spine J. 2018;8(6):586-592. doi:10.1177/2192568218757483

15. Tabaraee E, Ahn J, Bohl DD, Phillips FM, Singh K. Quantification of Multifidus Atrophy and Fatty Infiltration Following a Minimally Invasive Microdiscectomy. Int J Spine Surg. 2015;9:25. Published 2015 Jun 26. doi:10.14444/2025

16. Fuchs B, Weishaupt D, Zanetti M, Hodler J, Gerber C. Fatty degeneration of the muscles of the rotator cuff: assessment by computed tomography versus magnetic resonance imaging. J Shoulder Elbow Surg. 1999;8(6):599-605. doi:10.1016/s1058-2746(99)90097-6

17. Asil K, Yaldiz C. Retrospective Comparison of Radiological and Clinical Outcomes of PLIF and TLIF Techniques in Patients Who Underwent Lumbar Spinal Posterior Stabilization. Medicine (Baltimore). 2016;95(17):e3235. doi:10.1097/MD.0000000000003235

18. Lee DY, Jung TG, Lee SH. Single-level instrumented mini-open transforaminal lumbar interbody fusion in elderly patients. J Neurosurg Spine. 2008;9(2):137-144. doi:10.3171/SPI/2008/9/8/137

19. Bogduk N. Functional anatomy of the spine. Handb Clin Neurol. 2016;136:675-688. doi:10.1016/B978-0-444-53486-6.00032-6.

20. Phan K, Rao PJ, Kam AC, Mobbs RJ. Minimally invasive versus open transforaminal lumbar interbody fusion for treatment of degenerative lumbar disease: systematic review and meta-analysis. Eur Spine J. 2015;24(5):1017-1030. doi:10.1007/s00586-015-3903-4

21. Sari Y, Nakagami G, Kinoshita A, et al. Changes in serum and exudate creatine phosphokinase concentrations as an indicator of deep tissue injury: a pilot study. Int Wound J. 2008;5(5):674-680. doi:10.1111/j.1742-481X.2008.00543.x

22. Kawaguchi Y, Matsui $\mathrm{H}$, Tsuji H. Changes in serum creatine phosphokinase MM isoenzyme after lumbar spine surgery. Spine (Phila Pa 1976). 1997;22(9):1018-1023. doi:10.1097/00007632199705010-00015

23. Kim K, Isu T, Sugawara A, Matsumoto R, Isobe M. Comparison of the effect of 3 different approaches to the lumbar spinal canal on postoperative paraspinal muscle damage. Surg Neurol. 2008;69(2):109-113. 
24. Zhang D, Mao K, Qiang X. Comparing minimally invasive transforaminal lumbar interbody fusion and posterior lumbar interbody fusion for spondylolisthesis: A STROBE-compliant observational study. Medicine (Baltimore). 2017;96(37):e8011. doi:10.1097/MD.0000000000008011

25. Cheng WY, Zeng XX, Xiang X, Liu D, Zheng JP, Hu B. Zhongguo Gu Shang. 2019;32(10):965-970. doi:10.3969/j.issn.1003-0034.2019.10.019

26. Kim DY, Lee SH, Chung SK, Lee HY. Comparison of multifidus muscle atrophy and trunk extension muscle strength: percutaneous versus open pedicle screw fixation. Spine (Phila Pa 1976). 2005;30(1):123-129.

27. Junhui $L$, Zhengbao $P$, Wenbin $X$, et al. Comparison of pedicle fixation by the Wiltse approach and the conventional posterior open approach for thoracolumbar fractures, using MRI, histological and electrophysiological analyses of the multifidus muscle. Eur Spine J. 2017;26(5):1506-1514. doi:10.1007/s00586-017-5010-1

\section{Tables}

Table 1 Comparison of demographics among wiltse, MIS-TLIF and PLIF group

\begin{tabular}{ccccc}
\hline & wiltse & MIS-TLIF & PLIF & $P$ \\
\hline Gender & & & & 0.642 \\
male & 13 & 12 & 16 & \\
female & 16 & 16 & 14 & \\
Agelyears & $52.38 \pm 10.25$ & $49.54 \pm 10.78$ & $51.17 \pm 9.81$ & 0.580 \\
Surgical segment & & & & \\
L4-5 & 17 & 13 & 14 & \\
L5-1 & 12 & 15 & 16 & \\
Preoperative & & & & \\
Low back pain VAS & $3.17 \pm 2.42$ & $2.68 \pm 2.41$ & $2.80 \pm 2.17$ & 0.706 \\
Lower extremity pain VAS & $5.34 \pm 2.21$ & $4.96 \pm 2.25$ & $4.80 \pm 2.12$ & 0.623 \\
ODI score[\% & $63.46 \pm 11.36$ & $66.35 \pm 11.71$ & $64.22 \pm 12.21$ & 0.657 \\
JOA score & $12.21 \pm 5.34$ & $11.71 \pm 4.96$ & $11.70 \pm 5.21$ & 0.914 \\
\hline
\end{tabular}

wiltse: wiltse approach transforaminal lumbar interbody fusion; MIS-TLIF: minimally invasive transforaminal lumbar interbody fusion; PLIF: posterior lumbar interbody fusion.

Table 2 Comparison of Perioperative Metrics between wiltse, MIS-TLIF and PLIF group

\begin{tabular}{ccccc}
\hline & wiltse & MIS-TLIF & PLIF & $P$ \\
\hline Intraoperative bleeding(ml) & $241.38 \pm 98.26 \mathrm{a}$ & $164.29 \pm 65.06 \mathrm{~b}$ & $428.33 \pm 65.06 \mathrm{c}$ & $\square 0.001$ \\
Postoperative drainage (ml) & $127.24 \pm 41.99 \mathrm{a}$ & $53.93 \pm 25.44 \mathrm{~b}$ & $182.67 \pm 50.37 \mathrm{c}$ & $\square 0.001$ \\
Operation time (min) & $98.97 \pm 24.98 \mathrm{a}$ & $169.11 \pm 29.38 \mathrm{~b}$ & $94.67 \pm 25.43 \mathrm{a}$ & $\square 0.001$ \\
Fluoroscopy time (s) & $22.41 \pm 0.73 \mathrm{a}$ & $87.61 \pm 3.13 \mathrm{~b}$ & $22.41 \pm 0.73 \mathrm{a}$ & $\square 0.001$ \\
Postoperative bed time(d) & $3.3 \pm 0.48 \mathrm{a}$ & $2.68 \pm 0.71 \mathrm{~b}$ & $5.13 \pm 0.90 \mathrm{c}$ & $\square 0.001$ \\
\hline
\end{tabular}

LSD method was used to compare the statistical differences between groups.

wiltse: wiltse approach transforaminal lumbar interbody fusion; MIS-TLIF: minimally invasive transforaminal lumbar interbody fusion; PLIF: posterior lumbar interbody fusion. 
Table 3 CK(u/L) level of wiltse , MIS-TLIF and PLIF group

\begin{tabular}{|c|c|c|c|c|c|}
\hline & preoperative & 1 day & 3 days & 7 days & $P$ \\
\hline wiltse & $81.41 \pm 29.51 \mathrm{a}$ & $250.07 \pm 120.17 b$ & $112.31 \pm 58.52 \mathrm{c}$ & $83.79 \pm 27.65 a$ & प0.001* \\
\hline MIS-TLIF & $85.54 \pm 35.95 a$ & $244.75 \pm 100.26 b$ & $108.89 \pm 47.76 c$ & $78.39 \pm 51.04 \mathrm{a}$ & प0.001* \\
\hline PLIF & $87.93 \pm 32.00 \mathrm{a}$ & $414.10 \pm 247.49 \mathrm{~b}$ & $203.60 \pm 99.97 a$ & $89.30 \pm 23.76 a$ & प0.001* \\
\hline$P$ value & $0.736^{\&}$ & {$\left[0.001^{\&}\right.$} & {$\left[0.001^{\&}\right.$} & $0.515^{\&}$ & - \\
\hline
\end{tabular}

Bold indicates statistically significant values.

Multiple comparisons of $\mathrm{CK}$ at different time points using the Bonferroni post hoc test,and at least 1 identical subscript letter denoted no significant difference from each other at the 0.05 level.

*Repeated measurement analysis of variance

\&One-way analysis of variance

wiltse: wiltse approach transforaminal lumbar interbody fusion; MIS-TLIF: minimally invasive transforaminal lumbar interbody fusion; PLIF: posterior lumbar interbody fusion.

Table 4 VAS score of wiltse, MIS-TLIF and PLIF group

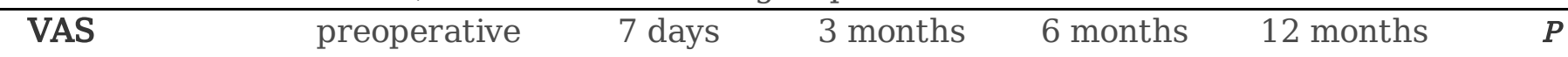

\begin{tabular}{ccccccc}
\hline Low back pain & & & & & \\
wiltse & $3.17 \pm 2.42 \mathrm{a}$ & $3.14 \pm 1.09 \mathrm{a}$ & $1.72 \pm 0.84 \mathrm{c}$ & $1.17 \pm 0.89 \mathrm{~d}$ & $0.86 \pm 0.74 \mathrm{e}$ & $\square 0.001^{*}$ \\
MIS-TLIF & $2.68 \pm 2.41 \mathrm{a}$ & $2.89 \pm 1.17 \mathrm{a}$ & $1.68 \pm 0.91 \mathrm{c}$ & $1.04 \pm 0.79 \mathrm{~d}$ & $0.79 \pm 0.63 \mathrm{e}$ & $\square 0.001^{*}$ \\
PLIF & $2.80 \pm 2.17 \mathrm{a}$ & $4.20 \pm 1.19 \mathrm{~b}$ & $2.77 \pm 0.90 \mathrm{a}$ & $2.17 \pm 0.79 \mathrm{c}$ & $1.93 \pm 0.83 \mathrm{~d}$ & $\square 0.001^{*}$ \\
P value & $0.706^{\&}$ & $\square 0.001^{\&}$ & $\square 0.001^{\&}$ & $\square 0.001^{\&}$ & $\square 0.001^{\&}$ & - \\
Lower limb pain & & & & & \\
wiltse & $5.34 \pm 2.21 \mathrm{a}$ & $2.07 \pm 0.96 \mathrm{~b}$ & $1.24 \pm 0.83 \mathrm{c}$ & $0.55 \pm 0.74 \mathrm{~d}$ & $0.41 \pm 0.63 \mathrm{e}$ & $\square 0.001^{*}$ \\
MIS-TLIF & $4.96 \pm 2.25 \mathrm{a}$ & $2.00 \pm 0.98 \mathrm{~b}$ & $1.25 \pm 0.89 \mathrm{c}$ & $0.68 \pm 0.72 \mathrm{~d}$ & $0.43 \pm 0.57 \mathrm{e}$ & $\square 0.001^{*}$ \\
PLIF & $4.80 \pm 2.12 \mathrm{a}$ & $1.80 \pm 1.03 \mathrm{~b}$ & $1.20 \pm 0.89 \mathrm{c}$ & $0.80 \pm 0.76 \mathrm{~d}$ & $0.47 \pm 0.57 \mathrm{e}$ & $\square 0.001^{*}$ \\
P value & $0.623^{\&}$ & $0.559^{\&}$ & $0.973^{\&}$ & $0.440^{\&}$ & $0.939^{\&}$ & - \\
\hline
\end{tabular}

Bold indicates statistically significant values.

Multiple comparisons of each variable at different time points using the Bonferroni post hoc test,and at least 1 identical subscript letter denoted no significant difference from each other at the 0.05 level.

*Repeated measurement analysis of variance

\&One-way analysis of variance

wiltse: wiltse approach transforaminal lumbar interbody fusion; MIS-TLIF: minimally invasive transforaminal lumbar interbody fusion; PLIF: posterior lumbar interbody fusion. 
Table 5 ODI and JOA score of wiltse, MIS-TLIF and PLIF group

\begin{tabular}{|c|c|c|c|c|c|}
\hline & preoperative & 3 months & 6 months & 12 months & $P$ \\
\hline \multicolumn{6}{|l|}{ ODI(\%) } \\
\hline wiltse & $63.46 \pm 11.36 \mathrm{a}$ & $24.67 \pm 4.34 \mathrm{~b}$ & $18.24 \pm 2.99 \mathrm{c}$ & $14.41 \pm 2.70 \mathrm{~d}$ & व0.001* \\
\hline MIS-TLIF & $66.35 \pm 11.71 \mathrm{a}$ & $23.89 \pm 4.67 b$ & $18.73 \pm 3.05 c$ & $13.96 \pm 3.02 \mathrm{~d}$ & [0.001* \\
\hline PLIF & $64.22 \pm 13.63 \mathrm{a}$ & $24.52 \pm 4.83 \mathrm{~b}$ & $20.30 \pm 3.58 c$ & $16.15 \pm 2.40 \mathrm{~d}$ & प0.001* \\
\hline $\mathrm{P}$ value & $0.657^{\&}$ & $0.795^{\&}$ & $00.042^{\mathcal{E}}$ & {$\left[0.007^{\mathcal{E}}\right.$} & - \\
\hline \multicolumn{6}{|l|}{ JOA score } \\
\hline wiltse & $12.21 \pm 5.34 \mathrm{a}$ & $23.00 \pm 2.20 \mathrm{~b}$ & $24.69 \pm 1.82 \mathrm{c}$ & $25.55 \pm 1.94 \mathrm{~d}$ & [0.001* \\
\hline MIS-TLIF & $11.71 \pm 4.96 \mathrm{a}$ & $23.14 \pm 2.40 \mathrm{~b}$ & $24.75 \pm 1.88 c$ & $25.43 \pm 2.38 d$ & प0.001* \\
\hline PLIF & $11.70 \pm 5.21 \mathrm{a}$ & $21.87 \pm 2.73 b$ & $23.57 \pm 2.29 c$ & $24.17 \pm 2.02 \mathrm{~d}$ & प0.001* \\
\hline $\mathrm{P}$ value & $0.914^{\&}$ & $0.098^{\&}$ & $0.044^{\&}$ & $0.025^{\mathcal{E}}$ & - \\
\hline
\end{tabular}

Bold indicates statistically significant values.

Multiple comparisons of each variable at different time points using the Bonferroni post hoc test, and at least 1 identical subscript letter denoted no significant difference from each other at the 0.05 level.

*Repeated measurement analysis of variance

\&One-way analysis of variance

wiltse: wiltse approach transforaminal lumbar interbody fusion; MIS-TLIF: minimally invasive transforaminal lumbar interbody fusion; PLIF: posterior lumbar interbody fusion.

Table 6 Comparison of Interbody fusion,multifidus atrophy and fatty infiltration among 3 groups

\begin{tabular}{ccccc}
\hline & wiltse & MIS-TLIF & PLIF & P \\
\hline Follow-up time(month) & & & & \\
CT & $12.14 \pm 2.78$ & $13.57 \pm 2.60$ & $12.73 \pm 2.80$ & 0.145 \\
MRI & $11.84 \pm 3.07$ & $12.25 \pm 2.70$ & $13.16 \pm 3.0$ & 0.528 \\
Interbody fusion rate & $89.7 \%$ & $92.9 \%$ & $93.3 \%$ & 0.854 \\
Ratio of multifidus atrophy & $24.13 \pm 6.82 \%$ a & $22.35 \pm 5.03 \%$ a & $41.70 \pm 8.84 \%$ b & $\square .001$ \\
Ratio of lean-to-total CSA & & & & \\
Preoperation & $69.28 \pm 8.11 \%$ & $70.64 \pm 7.92 \%$ & $68.17 \pm 7.09 \%$ & 0.476 \\
Last follow-up & $63.34 \pm 7.74 \% a$ & $64.03 \pm 7.19 \% a$ & $56.60 \pm 7.52 \%$ b & $\square 0.001$ \\
\hline
\end{tabular}

Bold indicates statistically significant values.

LSD method was used to compare the statistical differences between groups and at least 1 identical subscript letter denoted no significant difference from each other at the 0.05 level.

wiltse: wiltse approach transforaminal lumbar interbody fusion; MIS-TLIF: minimally invasive transforaminal lumbar interbody fusion; PLIF: posterior lumbar interbody fusion.

\section{Figures}




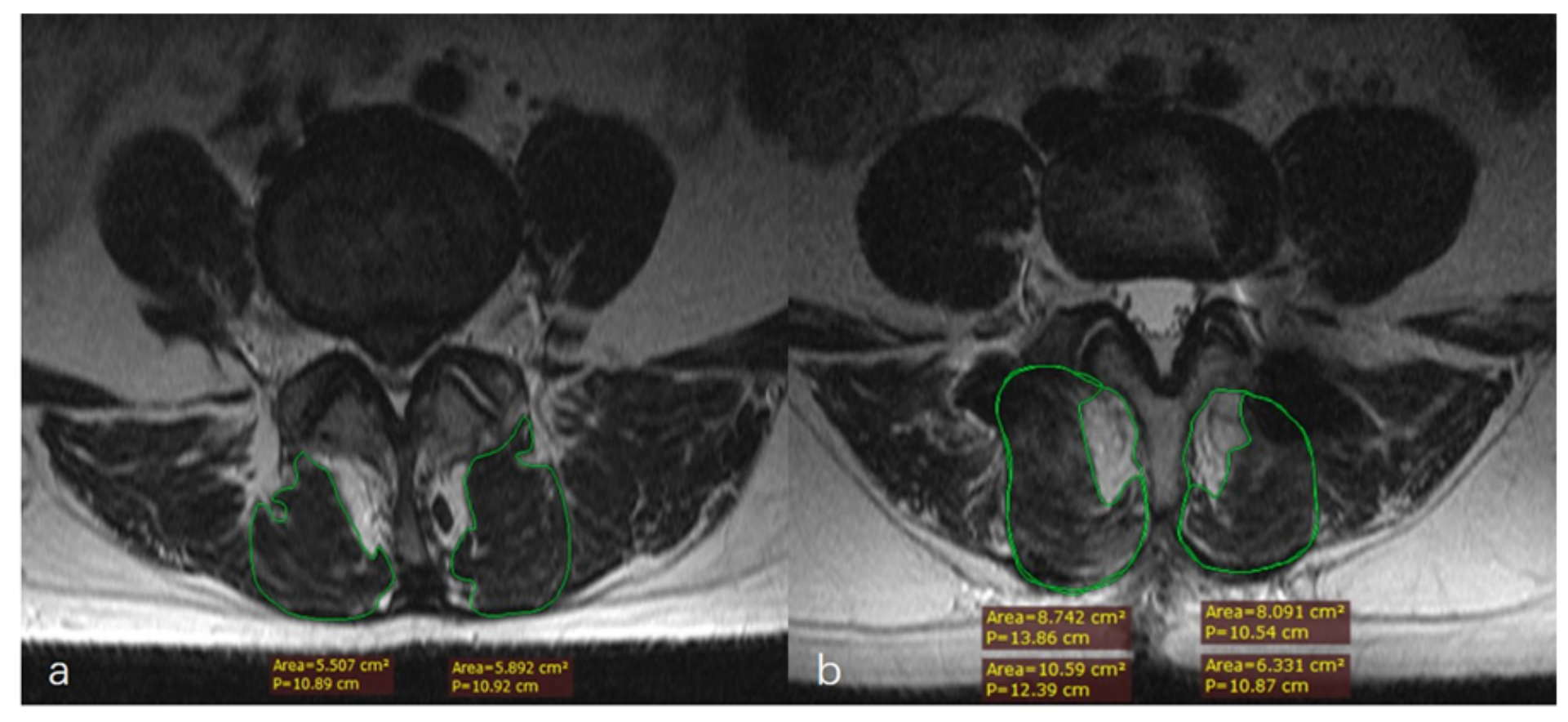

Figure 1

\a冈Preoperative axial T2-weighted MRI image demonstrates lean cross-sectional area measurements (b) Postoperative axial T2-weighted MRI image demonstrates total and lean cross-sectional measurements
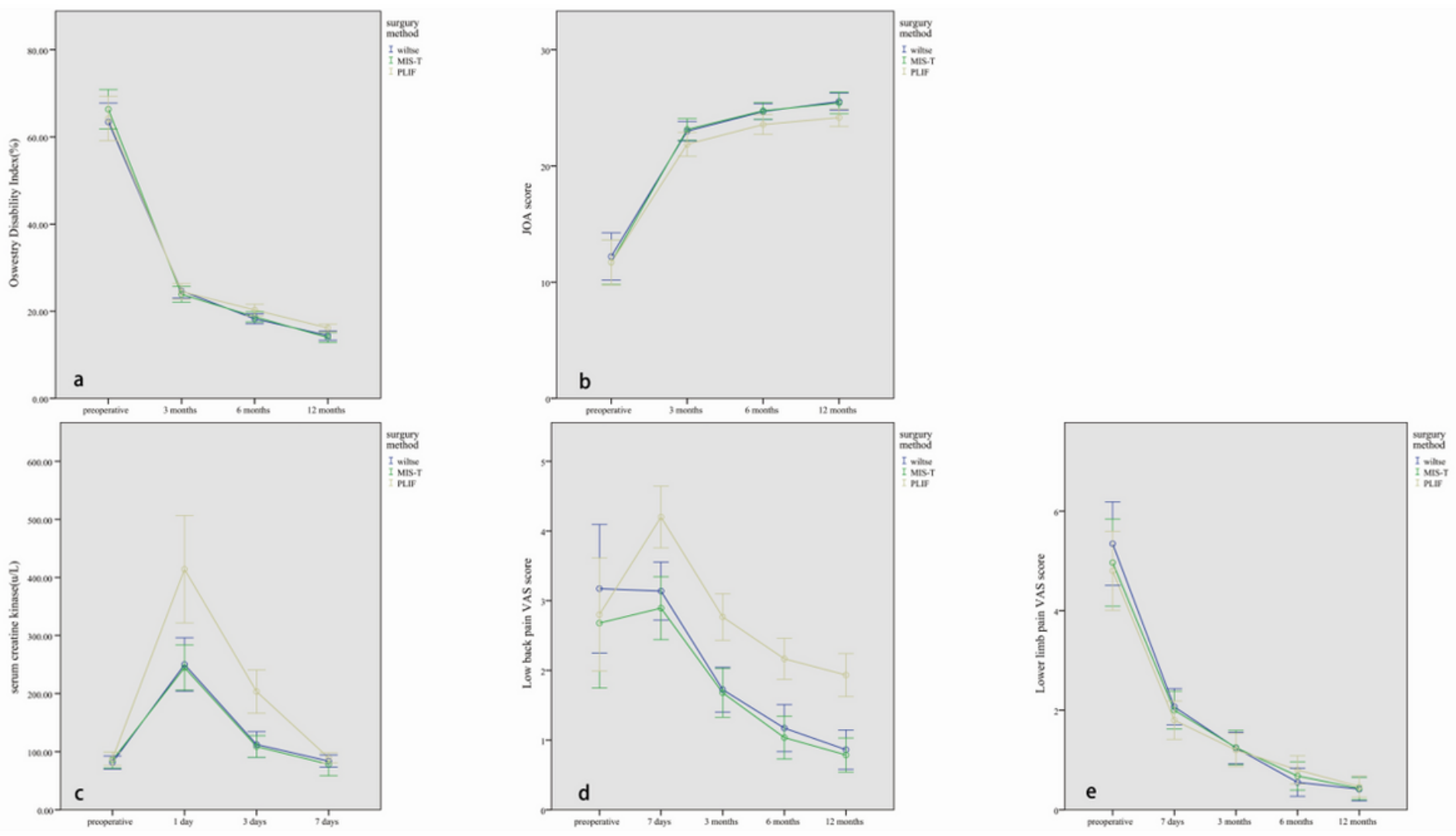

Figure 2 
(a) ODI of wiltse,MIS-TLIF group at 6 and 12months after surgery were lower than PLIF (b) JOA score of wiltse,MIS-TLIF group at 6 and 12months after surgery were higher than PLIF group: (c) serum creatine kinase level of wiltse, MIS-TLIF group on 1 and 3day(s) after surgery were significantly lower than PLIF group (d) low back pain VAS score of wiltse , MIS-TLIF group at 7 days, 3 months, 6 months , 12 months after surgery were significantly lower than PLIF group (e) lower limb pain VAS score of wiltse, MIS-TLIF and PLIF group showed no statistical difference
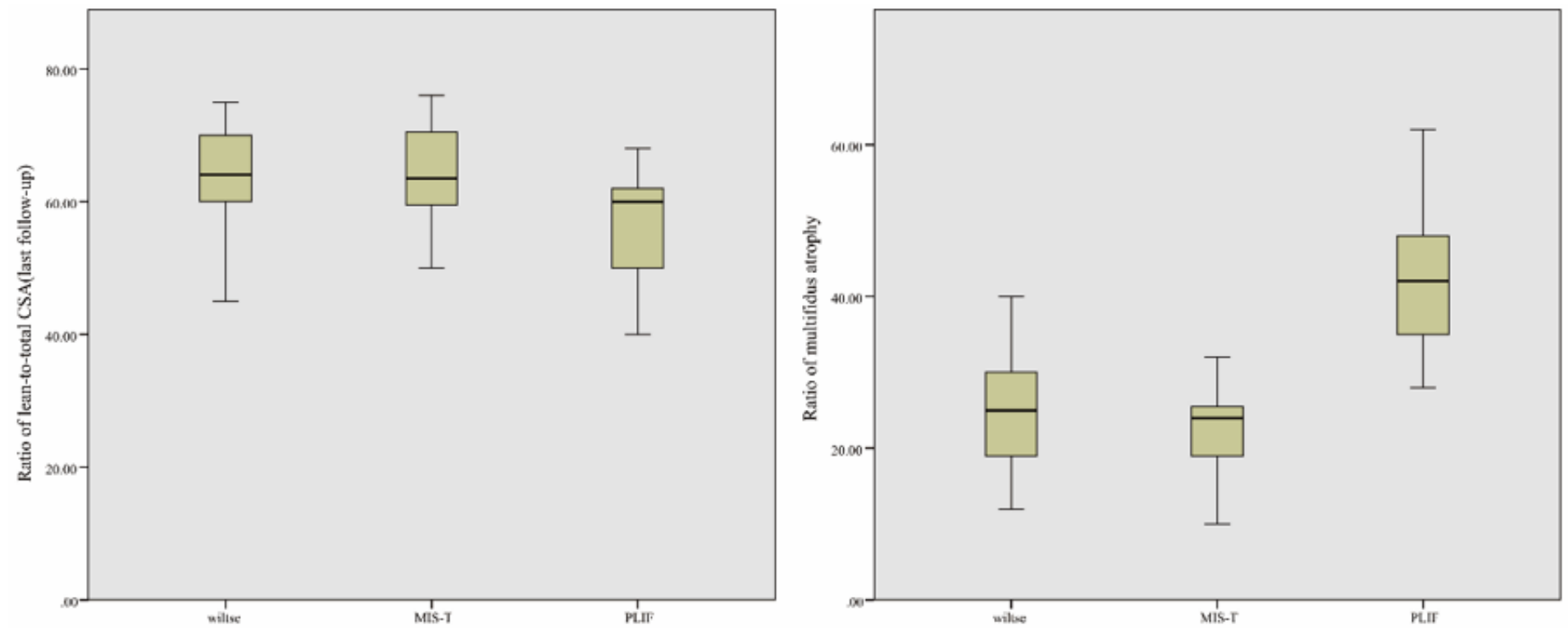

\section{Figure 3}

Ratio of lean-to-total CSA :PLIF group $(56.60 \pm 7.52 \%)$ was lower than in wiltse group $(63.34 \pm 7.74 \%)$ and MIS-TLIF group( $64.03 \pm 7.19 \%)$ Ratio of multifidus atrophy: PLIF group $(41.70 \pm 8.84) \%$ was significantly higher than that in the wiltse group $(24.13 \pm 6.82) \%$ and the MIS-TILF group $(22.35 \pm 5.03) \%$
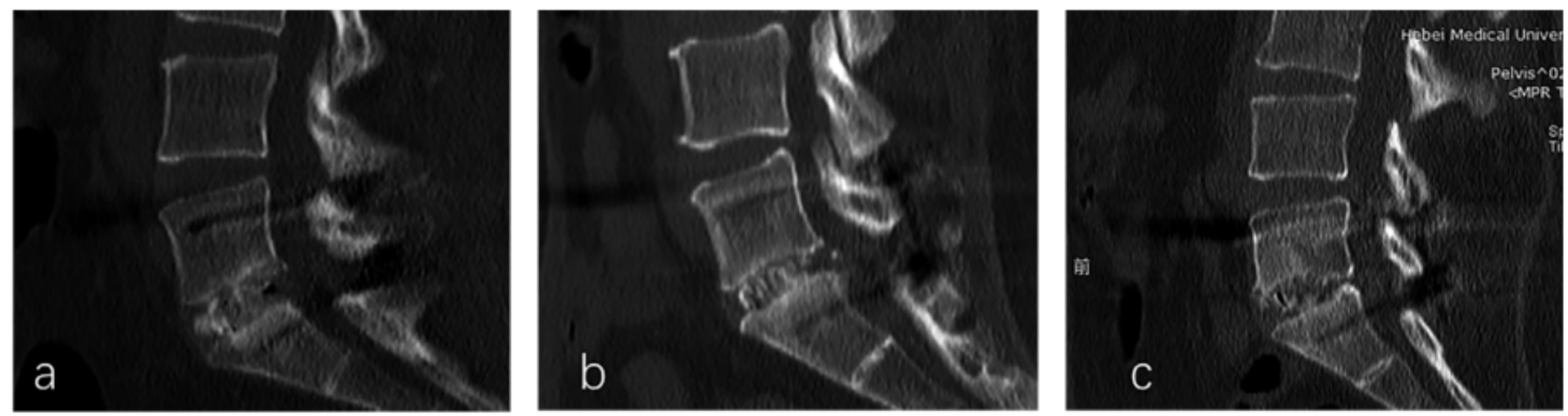

\section{Figure 4}

wiltse, MIS-TLIF and PLIF group showed definite bone connection (a)12 months CT of a wiltse approach TLIF patient (b)14 months CT of a MIS-TLIF patient (c)36 months CT of a PLIF patient 

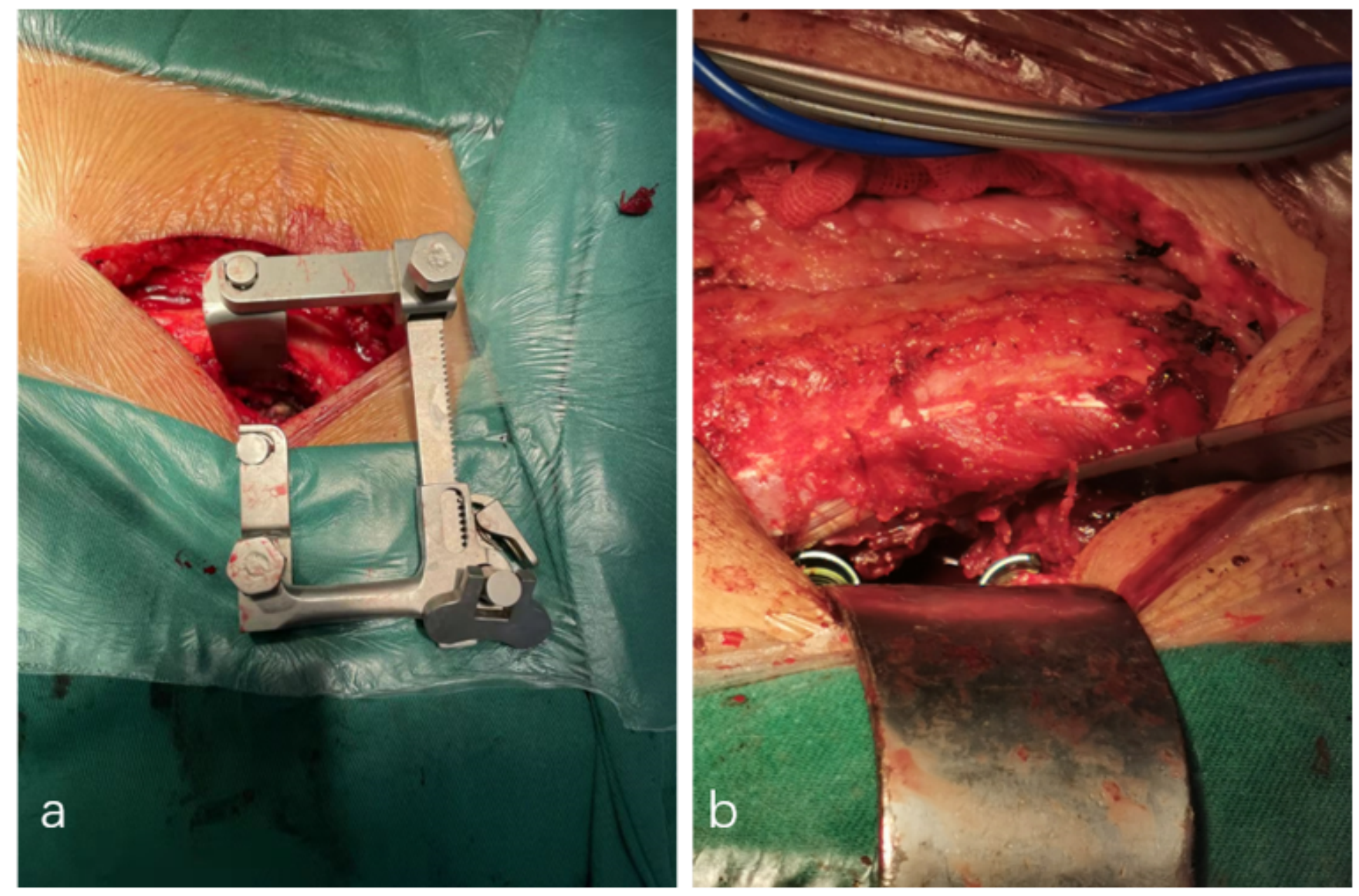

\section{Figure 5}

(a) Show wiltse approach TLIF assisted by the new-type retractor,the anatomical structure can be identified easily and decompression and fusion becomes more intuitive and convenient (b)show a traditional wiltse approach TLIF surgery.It requires more energy of assistant and is still hard to expose especially in obese or muscular patients. 

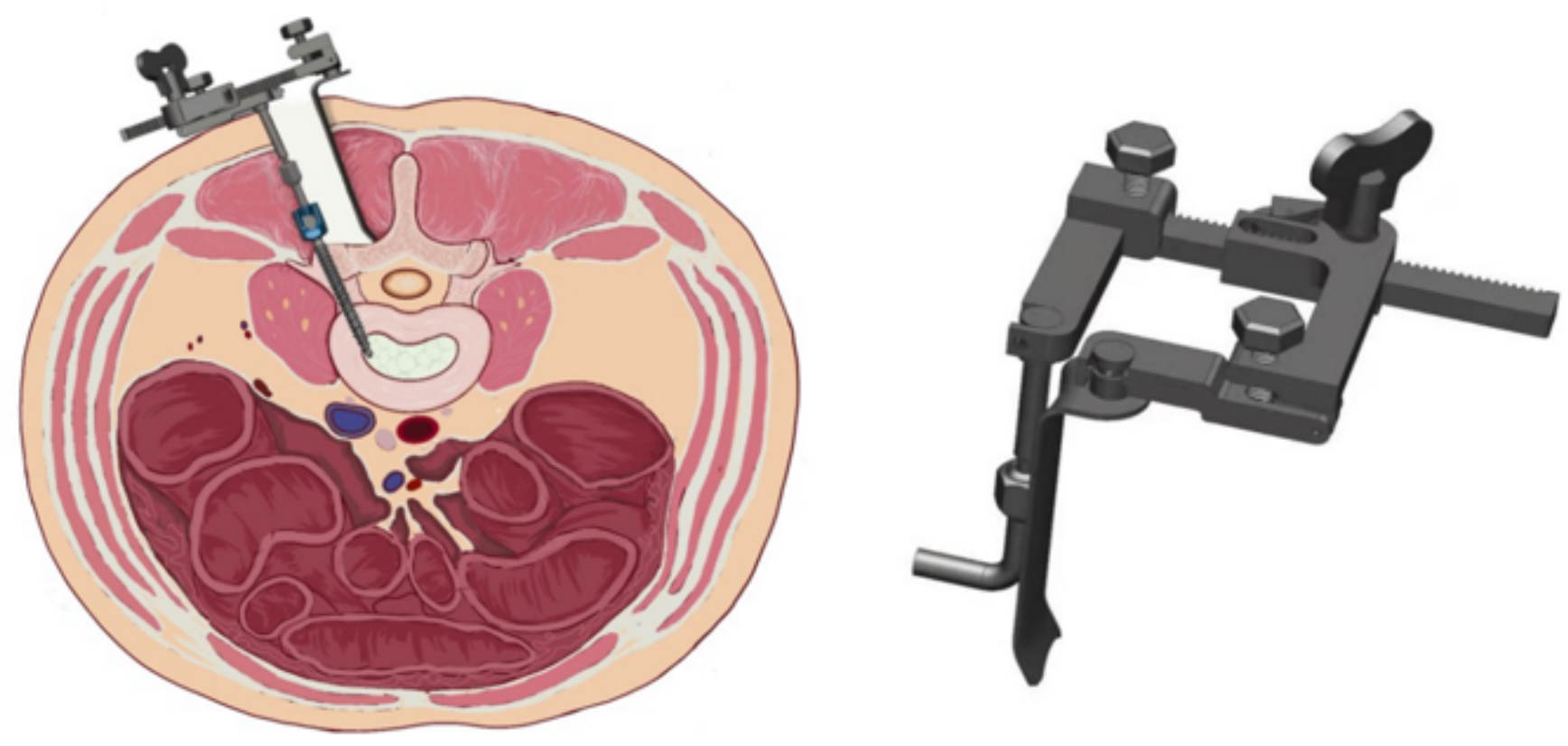

Figure 6

Show the new type retractor for wiltse approach TLIF. After pedicle screw implantation, the retractor can be fixed on the pedicle screw. The rod attached to the pedicle screw pulls the muscle laterally, and the separation blade pulls the muscle medially. The distance and degree of retraction can be adjusted by rotating the knob. 


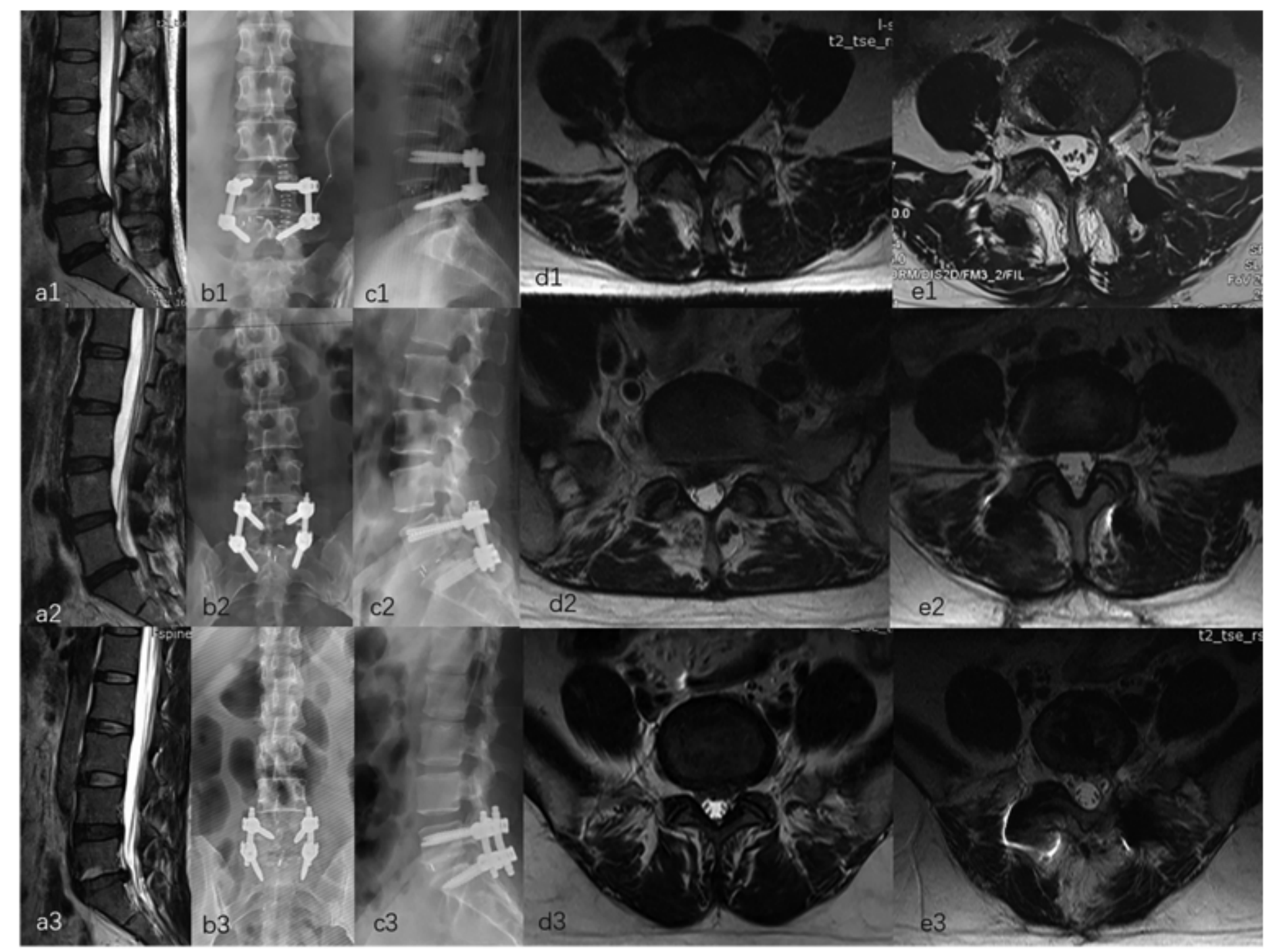

\section{Figure 7}

(a1-e1)1 case of wiltse approach TLIF patient (a2-e2)1 case of MIS-TLIF patient (a3-e3) 1 case of PLIF patient (a1)MRI before surgery showed disc herniation in L4-L5 segment (b1 and c1) postoperative anterior and lateral DR (d1) axial T2 MRI show disc herniation and multifidus before surgery (e1) axial T2 MRI show surgery level multifidus at last follow-up after surgery with no significant atrophy and fatty infiltration (a2):MRI before surgery showed disc herniation in L5-S1 segment (b2 and c2) postoperative anterior and lateral DR (d2) axial T2 MRI show disc herniation and multifidus before surgery (e2) axial T2 MRI show surgery level multifidus at last follow-up after surgery with no significant atrophy and fatty infiltration (a3):MRI before surgery showed disc herniation in L5-S1 segment (b3 and c3) postoperative anterior and lateral DR (d3) axial T2 MRI show disc herniation and multifidus before surgery (e3) axial T2 MRI show surgery level multifidus at last follow-up after surgery with significant atrophy and fatty infiltration 